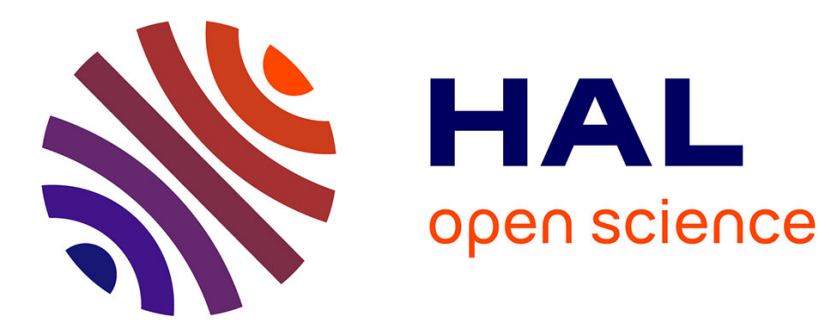

\title{
Non-Separable Preferences do not Rule Out Aggregate Instability under Balanced-Budget Rules: A Note
}

\author{
Nicolas Abad, Thomas Seegmuller, Alain Venditti
}

\section{To cite this version:}

Nicolas Abad, Thomas Seegmuller, Alain Venditti. Non-Separable Preferences do not Rule Out Aggregate Instability under Balanced-Budget Rules: A Note. 2014. halshs-01933532

\author{
HAL Id: halshs-01933532 \\ https://shs.hal.science/halshs-01933532 \\ Preprint submitted on 23 Nov 2018
}

HAL is a multi-disciplinary open access archive for the deposit and dissemination of scientific research documents, whether they are published or not. The documents may come from teaching and research institutions in France or abroad, or from public or private research centers.
L'archive ouverte pluridisciplinaire HAL, est destinée au dépôt et à la diffusion de documents scientifiques de niveau recherche, publiés ou non, émanant des établissements d'enseignement et de recherche français ou étrangers, des laboratoires publics ou privés. 


\section{amse}

école d'économie d'aix-marseille

aix-marseille school of economics

\section{Working Papers / Documents de travail}

Non-Separable Preferences do not Rule Out Aggregate Instability under Balanced-Budget Rules: A Note

Nicolas Abad

Thomas Seegmuller

Alain Venditti 


\title{
Non-Separable Preferences do not Rule Out
}

\section{Aggregate Instability under Balanced-Budget}

\author{
Rules: A Note* \\ Nicolas ABAD \\ Aix-Marseille Univ., CNRS, EHESS, Centrale Marseille, AMSE, France. Email: \\ nicolas_abad@hotmail.fr \\ Thomas SEEGMULLER \\ Aix-Marseille Univ., CNRS, EHESS, Centrale Marseille, AMSE, France. Email: \\ thomas.seegmuller@univ-amu.fr
}

\begin{abstract}
Alain VENDITTI
Aix-Marseille Univ., CNRS, EHESS, Centrale Marseille, AMSE \&

EDHEC Business School, France. Email: alain.venditti@univ-amu.fr
\end{abstract}

First version: April 2012; First Revision: February 2014; Last Revision: November 2014

\footnotetext{
*We thank two anonymous referees and an Associate Editor together with Stefano Bosi, Raouf Boucekkine, Hippolyte d'Albis, Jean-Michel Grandmont, Cuong Le Van, Carine Nourry, Xavier Raurich, Chrissy Giannitsarou and Roger Farmer for useful comments and suggestions. This paper also benefited from presentations at the "Conference in honor of Cuong Le Van", MSE-PSE-University of Paris 1, Paris, December 2011; OLG Days 2012, Marseille; PET 2012, Taipei and LAGV 2012, Marseille.
} 
Proposed running head: Instability under Balanced-Budget Rules

Corresponding author: Alain Venditti, GREQAM, 2 rue de la Charité, 13002 Marseille, France. Phone: 33-491317496. Fax: 33-491900227. Email: alain.venditti@univ-amu.fr 


\begin{abstract}
We investigate the role of non-separable preferences on the occurrence of macroeconomic instability under a balanced-budget rule where government spending is financed by a tax on labor income. Considering a one-sector neoclassical growth model with a large class of non-separable utility functions, we find that expectationsdriven fluctuations easily occur when consumption and labor are Edgeworth substitutes or weak Edgeworth complements. Under these properties, an intermediate range of tax rates and a sufficiently low elasticity of intertemporal substitution in consumption lead to instability.
\end{abstract}

Keywords: Indeterminacy, expectations-driven business cycles, labor income taxes, balanced-budget rule, non-separable preferences. 


\section{Introduction}

According to Schaechter et al. (2012), around 60 countries, mostly advanced, have adopted some kind of balanced-budget rule either at the national or supra-national level in 2012. Balanced-budget rules can be specified as overall balance, structural or cyclically adjusted balance, and balance "over the cycle."

Since the beginning of the Great Recession in 2008, the first type of rule has been widely discussed in particular in Europe and adopted by some countries like Germany or Switzerland as a "Golden Rule". But this type of balancedbudget rule does not have economic stabilization features. Following standard Keynesian arguments, such balanced-budget rules are not worthwhile as they amplify business cycles. During booms, government cuts taxes and sets a higher public spending, while in recessions, taxes are increased and such fiscal policies contract the aggregate demand.

An additional argument along a similar line has been stressed in the seminal contribution of Schmitt-Grohé and Uribe (1997) (SGU). They show that a balanced-budget rule may be a source of aggregate instability as it may generate a strong volatility of agents' expectations. In a standard Ramsey model with a constant stream of government expenditures financed by a distortionary tax on labor income, any increase in the expected tax rate implies a reduction in future labor supply and therefore of capital returns. Current investment 
decreases so that households work less. Under a balanced-budget, the tax rate is decreasing in the tax base. Facing a decreasing labor income, the government then has to increase the tax rate to maintain the budget balanced and expectations are self-fulfilling. Further contributions, such as Guo and Lansing (1998), show that a progressive income tax stabilizes the economy by ruling-out expectations-driven fluctuations generated by productive externalities. Considering instead constant income taxes on capital and labor income financing an endogenously determined government spending, Guo and Harrison (2004) show that aggregate instability never occurs. Hence, these contributions confirm that the destabilizing mechanism of balanced-budget rules strongly relies on the regressive property of the labor income tax.

The main conclusions of SGU are established under the assumption of additively-separable preferences with infinitely elastic labor and a CobbDouglas technology. In a subsequent work, Linnemann (2008) shows however that the destabilizing effect of balanced-budget rules is not robust to the consideration of non-separable preferences. He considers the non-separable specification of King-Plosser-Rebelo (1988) (KPR) which is compatible with balanced growth and constant labor, and he shows that when the tax rate on wage income is calibrated to match realistic values, expectations-driven fluctuations and aggregate instability are ruled out. However, Linneman (2008) introduces in his analysis an additional restriction that strongly limits the set of 
KPR preferences he considers. He assumes that the elasticity of intertemporal substitution in consumption is lower than one which implies that consumption and labor are strong Edgeworth complements.

In this paper, our aim is to prove that as long as a sufficiently large set of non-separable preferences is considered, the destabilizing effect of balancedbudget rules under labor-income taxes exhibited by SGU is a robust property. We prove that aggregate instability under balanced-budget rules can be easily obtained with non-separable preferences commonly used in the macroeconomic literature, provided that the Edgeworth-complementarity between consumption and labor is not too strong. This is explored in a neoclassical infinitehorizon growth model embedding most popular non-separable preferences used by macroeconomists. We consider two classes of non-separable utility functions: i) a linearly homogeneous specification, ii) the Jaimovich-Rebelo (2008) (JR) specification where the degree of income effect can be controlled and which admits as polar cases the Greenwood-Hercowitz-Huffman (1988) (GHH) utility function (with no income effect) and the KPR formulation (with a maximal degree of income effect). A generalized production function describes the technology of the firms in order to cover a large set of substitutability between capital and labor. ${ }^{1}$ The government is characterized by the same balanced-

\footnotetext{
${ }^{1}$ Under the same additively-separable preferences as in SGU, Ghilardi and Rossi (2014) already depart from the Cobb-Douglas technology considering a CES production function
} 
budget rule considered by SGU for which the tax rate is counter-cyclical with respect to the tax base.

We prove that expectations-driven fluctuations due to a labor income tax and a balanced-budget rule is a robust outcome under non-separable preferences. More precisely, local indeterminacy is likely to occur with preferences characterized by either Edgeworth-substitutability or a low enough Edgeworthcomplementarity between consumption and labor. These properties are always satisfied if the utility function is linearly homogeneous, but require a large enough degree of income effect for JR preferences. We numerically illustrate our results for each preferences under a standard parametrization based on quaterly data. We show that the likelihood of indeterminacy increases as labor and consumption exhibit more Edgeworth-substitutability and that a large set of OECD countries may experience aggregate instability under plausible parametrizations.

The rest of the paper is organized as follows. In the next section, we present the model while in Section 3, we prove the existence of a normalized steady state. In Section 4, we derive our main results and provide numerical illustrations in Section 5. The economic interpretations are discussed in Section 6. Section 7 concludes and Section 8 presents the proofs of our main results while the proof of technical results are given in a Technical Appendix available and show that aggregate instability is less likely when capital and labor are weak substitutes. 
upon request.

\section{The model}

We consider an infinite-horizon model with three types of agents, consumers, producers and the government which fixes labor income taxes according to a balanced-budget rule.

\subsection{Government}

As in SGU, we assume that the government chooses a constant level of public spending $G$, that neither affects the preferences nor the technology, following a balanced-budget rule. The government expenditure is equal to the total tax revenue $\Omega(t)$ generated by a tax rate, $\tau(t)$, applied on labor income, $w(t) l(t)$, with $w(t)$ the wage rate and $l(t)$ the labor supplied:

$$
G=\Omega(t)=\tau(t) w(t) l(t)
$$

The balanced-budget rule can be written as:

$$
\tau(t)=\frac{G}{w(t) l(t)}
$$

Since the government spending is constant, the tax rate decreases with respect to its tax base.

Three main arguments can be used to justify our formulation. First, some type of balanced-budget rule, either at the national or supra-national 
level, have been recently adopted by many countries, mostly advanced (see Schaechter et al. (2012)). Second, taxes on capital income are usually quite flat and thus not adjusted along the business cycle. Third, as shown by Lane (2003), the government spending is counter-cyclical with respect to GDP in most OECD countries. As already discussed by SGU and the literature mentioned above, the likelihood of expectations-driven fluctuations increases as the labor income tax is more regressive. It is easy to check that this regressivity is reinforced if we include a constant tax on capital income and/or a countercyclical government spending in the rule (2). Because the scope of this paper is to investigate the role of non-separable preferences on the main mechanism leading to indeterminacy, we focus on the simple rule considered in SGU.

\subsection{Households}

We consider an economy populated by a large number of identical infinitelylived agents. We assume without loss of generality that population is constant and normalized to one. At each point in time, a representative agent supplies elastically an amount of labor $l \in[0, \bar{l}]$, with $\bar{l}>1$ his time endowment. He

then derives utility from consumption $c$ and leisure $\mathcal{L}=\bar{l}-l$ according to the instantaneous utility function $U(c, \mathcal{L} / B)$, where $B$ is a constant scaling parameter, which satisfies: 
Assumption 1. $U(c, \mathcal{L} / B)$ is twice differentiable, increasing with respect to each argument and concave. Moreover, $\frac{U_{c \mathcal{L}}}{U_{c}} \frac{\mathcal{L}}{B}-\frac{U_{\mathcal{L} \mathcal{L}}}{U_{\mathcal{L}}} \frac{\mathcal{L}}{B} \neq 1$, $\lim _{X \rightarrow 0} X U_{X}(c, X) / U_{c}(c, X)=0$ and $\lim _{X \rightarrow+\infty} X U_{X}(c, X) / U_{c}(c, X)=+\infty$, or $\lim _{X \rightarrow 0} X U_{X}(c, X) / U_{c}(c, X)=+\infty$ and $\lim _{X \rightarrow+\infty} X U_{X}(c, X) / U_{c}(c, X)=$ 0.

This assumption is a sufficient condition for the existence of a normalized steady state. Within the class of utility functions satisfying these properties, we consider two different specifications of non-separable preferences commonly used in the literature:

i) A linearly homogeneous utility function $U(c, \mathcal{L} / B)$ characterized by the share of consumption within total utility $\alpha(c, \mathcal{L} / B) \in(0,1)$ defined by:

$$
\alpha(c, \mathcal{L} / B)=\frac{U_{c}(c, \mathcal{L} / B) c}{U(c, \mathcal{L} / B)}
$$

while the share of leisure is given by $1-\alpha(c, \mathcal{L} / B)$. As an illustration of such a function we have the CES specification:

$$
U(c, \mathcal{L} / B)=\left[\alpha c^{\phi}+(1-\alpha)(\mathcal{L} / B)^{\phi}\right]^{\frac{1}{\phi}}
$$

with $\phi<1$ and $1 /(1-\phi)$ is the elasticity of substitution between consumption and leisure. We derive in this case $\alpha(c, \mathcal{L} / B)={\frac{\alpha c^{\phi}}{\left[\alpha c^{\phi}+(1-\alpha)(\mathcal{L} / B)^{\phi}\right]}}^{2}$

\footnotetext{
${ }^{2}$ Note that Assumption 1 is not satisfied in the Cobb-Douglas case with $\phi=0$. However, the existence of a steady state can be analytically proved by using directly the linear relationship between consumption and leisure in the first order conditions.
} 
ii) A Jaimovich-Rebelo (2008) formulation such that

$$
U(c, \mathcal{L} / B)=\frac{\left(c+(\mathcal{L} / B)^{1+\chi_{c}}\right)^{1-\theta}}{1-\theta}
$$

with $\theta, \chi>0$ and $\gamma \in[0,1]$. The parameter $\gamma$ controls the degree of income effect which allows to encompass two standard formulations. On the one hand, in absence of income effect $(\gamma=0)$, the $\mathrm{GHH}$ formulation is obtained and yields a labor supply independent of consumption. On the other hand, when the income effect is the largest $(\gamma=1)$, the utility function corresponds to the KPR formulation which is compatible with balanced growth and stationary hours worked.

Note that these utility functions satisfy normality of consumption and labor. In addition, the JR specification requires additional restrictions to satisfy the concavity conditions when $\gamma \neq 0$ (see Section 4.2 for further details). We also introduce the definition of Edgeworth-substitutability between consumption and labor:

Definition 1. If the marginal utility of consumption is increasing in leisure such that $U_{c \mathcal{L}}(c, \mathcal{L} / B)>0$, then consumption and labor are Edgeworth substitutes. Otherwise, if $U_{c \mathcal{L}}(c, \mathcal{L} / B)<0$, consumption and labor are Edgeworth complements.

We conclude that with linearly homogeneous preferences, consumption and la- 
bor are always Edgeworth substitutes since $U_{c \mathcal{L}}(c, \mathcal{L} / B)>0$, but with JR preferences Edgeworth-substitutability between consumption and labor requires $\gamma>\theta$ while Edgeworth-complementarity is obtained when $\gamma<\theta$. Note that Linnemann (2008) restricts his attention to a KPR utility function with $\theta>1$ which immediately implies strong Edgeworth-complementarity between consumption and labor.

The maximization program of the representative agent is given by:

$$
\begin{array}{cl}
\max _{c(t), l(t), K(t)} & \int_{t=0}^{+\infty} e^{-\rho t} U(c(t),(\bar{l}-l(t)) / B) \\
\text { s.t. } & c(t)+\dot{K}(t)+\delta K(t)=r(t) K(t)+(1-\tau(t)) w(t) l(t) \\
& K(0)=K_{0}>0 \text { given }
\end{array}
$$

where $r(t)$ is the rental rate of capital, $\rho>0$ the discount rate, $K(t)$ the capital stock and $\delta>0$ the depreciation rate of capital. Moreover, we assume that each household considers as given the tax rate $\tau(t)$. Let us introduce the Hamiltonian in current value:

$$
\mathcal{H}=U(c(t),(\bar{l}-l(t)) / B)+\lambda(t)[r(t) K(t)+(1-\tau(t)) w(t) l(t)-c(t)-\delta K(t)]
$$

with $\lambda(t)$ the shadow price of capital $K(t)$. Considering the prices (10)-(11) and the tax rate $\tau(t)$ as given, we derive the following first order conditions:

$$
\begin{array}{r}
U_{c}(c(t),(\bar{l}-l(t)) / B)=\lambda(t) \\
(1 / B) U_{\mathcal{L}}(c(t),(\bar{l}-l(t)) / B)=\lambda(t)(1-\tau(t)) w(t) \\
\dot{\lambda}(t)=-\lambda(t)[r(t)-\rho-\delta]
\end{array}
$$


Any solution with $K(0)=K_{0}$ should also satisfy the transversality condition:

$$
\lim _{t \rightarrow+\infty} e^{-\rho t} \lambda(t) K(t)=0
$$

\section{$2.3 \quad$ Firms}

We consider a competitive economy with a continuum of firms of unit size who produce a single good $Y$. Firms use capital $K$ and labor $l$ through a constant returns to scale production function $Y=A F(K, l)$, with $A$ a scaling parameter. Let $a=K / l$ so that the production function writes $Y / l=A f(a)$.

Assumption 2. $f(a)$ is twice differentiable, increasing and concave.

From profit maximization we obtain the wage rate $w(t)$ and the rental rate of capital $r(t)$ as:

$$
\begin{aligned}
r(t) & =A f^{\prime}(a(t)) \\
w(t) & =A\left[f(a(t))-a(t) f^{\prime}(a(t))\right]
\end{aligned}
$$

We also compute the share of capital in total income:

$$
s(a)=\frac{a f^{\prime}(a)}{f(a)} \in(0,1)
$$

and the elasticity of capital-labor substitution:

$$
\sigma(a)=-\frac{(1-s(a)) f^{\prime}(a)}{a f^{\prime \prime}(a)}>0
$$

Our formulation admits as particular cases the technologies with a constant elasticity of capital-labor substitution such as the Cobb-Douglas function with 
$\sigma(a)=1$ or the CES specification with $\sigma(a)=\sigma \neq 1$ for all $a>0$. As a result, our model encompasses the formulation of Ghilardi and Rossi (2014) where the results of SGU are extended to a CES technology. We introduce the following restriction:

Assumption 3. Capital and labor are sufficiently substitutes with $\sigma(a)>$ $s(a)$.

This ensures that labor income is increasing with respect to the amount of labor.

\section{Equilibrium}

The following system of differential equations in $K$ and $\lambda$, the shadow price of capital, describes the dynamics of the economy: ${ }^{3}$

$$
\begin{aligned}
\dot{K}(t) & =r(K(t), \lambda(t)) K(t)+(1-\tau(K(t), \lambda(t))) w(K(t), \lambda(t)) l(K(t), \lambda(t)) \\
& -\delta K(t)-c(K(t), \lambda(t)) \\
\dot{\lambda}(t) & =-\lambda(t)[r(K(t), \lambda(t))-\rho-\delta]
\end{aligned}
$$

An intertemporal equilibrium is then a path $\{K(t), \lambda(t)\}_{t \geq 0}$, with $K(0)=K_{0}$, that satisfies equations (14) and the transversality condition (9).

\footnotetext{
${ }^{3}$ For details, the interested reader can refer to Section 1 of a Technical Appendix available upon request.
} 
A steady state is a solution $\left(a^{*}, l^{*}, c^{*}, \tau^{*}\right)$, with $a^{*}=K^{*} / l^{*}$, that satisfies the balanced-budget rule (2), equations (10)-(11), the first-order conditions of the households' program (6)-(8) and equations (14) with $\dot{K}=\dot{\lambda}=0$. We use the scaling parameters $A$ and $B$ to ensure the existence of a normalized steady state (NSS), $a^{*}=1$ and $l^{*}=1$, which remains invariant with respect to preference and technological parameters.

Proposition 1. Let Assumptions 1-2 hold. Then there exist unique values $A^{*}$ and $B^{*}$ such that when $A=A^{*}$ and $B=B^{*},\left(a^{*}, l^{*}\right)=(1,1)$ is a NSS.

Proof: See Section 2 of the Technical Appendix.

Let us introduce the following elasticities:

$$
\varepsilon_{c c}=-\frac{U_{c}(c, \mathcal{L})}{U_{c c}(c, \mathcal{L}) c}, \quad \varepsilon_{l c}=-\frac{U_{\mathcal{L}}(c, \mathcal{L})}{U_{\mathcal{L} c}(c, \mathcal{L}) c}, \quad \varepsilon_{c l}=-\frac{U_{c}(c, \mathcal{L})}{U_{c \mathcal{L}}(c, \mathcal{L}) l}, \quad \varepsilon_{l l}=-\frac{U_{\mathcal{L}}(c, \mathcal{L})}{U_{\mathcal{L L}}(c, \mathcal{L}) l}
$$

Normality of consumption and leisure requires $\frac{1}{\varepsilon_{c c}}-\frac{1}{\varepsilon_{l c}} \geq 0$ and $\frac{1}{\varepsilon_{l l}}-\frac{1}{\varepsilon_{c l}} \geq 0$ and holds for both utility functions we consider. Concavity requires $\frac{1}{\varepsilon_{c c}} \frac{1}{\varepsilon_{l l}}-\frac{1}{\varepsilon_{c l}} \frac{1}{\varepsilon_{l c}} \geq$ 0. This property is satisfied for linearly homogeneous preferences, but the JR formulation requires further restrictions in the neighboorhood of the NSS (see proof of Proposition 3). According to Definition 1, note that when $\varepsilon_{c l}$ and $\varepsilon_{l c}$ are negative (positive), consumption and labor are Edgeworth substitutes (complements).

In the rest of the paper, we evaluate all the shares and elasticities previously 
defined at the NSS. From (3), (12) and (13), we denote indeed $\alpha\left(c^{*},(\bar{l}-\right.$ 1) $\left./ B^{*}\right)=\alpha, s(1)=s$ and $\sigma(1)=\sigma$.

\section{Instability with non-separable preferences}

This section provides simple conditions for the existence of local indeterminacy with non-separable preferences. Using the trace and the determinant of the Jacobian matrix associated to the linearized dynamic system evaluated at the NSS, we immediately derive a necessary condition for the existence of indeterminacy whatever the specification of the utility function:

Lemma 1. Let Assumptions 1-3 hold. A necessary condition for local indeterminacy of the NSS is $\tau>\underline{\tau}$, with:

$$
\underline{\tau}=\frac{\frac{s}{\sigma}+\varepsilon_{c c}\left(\frac{1}{\varepsilon_{c c}} \frac{1}{\varepsilon_{l l}}-\frac{1}{\varepsilon_{c l}} \frac{1}{\varepsilon_{l c}}\right)}{1+\varepsilon_{c c}\left(\frac{1}{\varepsilon_{c c}} \frac{1}{\varepsilon_{l l}}-\frac{1}{\varepsilon_{c l}} \frac{1}{\varepsilon_{l c}}\right)}
$$

Proof: See Section 3 of the Technical Appendix.

Given that the term $\frac{1}{\varepsilon_{c c}} \frac{1}{\varepsilon_{l l}}-\frac{1}{\varepsilon_{c l}} \frac{1}{\varepsilon_{l c}}$ measures the degree of concavity of the utility function, we conclude that stronger degrees of concavity imply that indeterminacy requires higher tax rates on labor income. Moreover, for any given specification of preferences, the lower bound $\underline{\tau}$ increases with the capital share of income and decreases with the elasticity of capital-labor substitution. In the particular case of additively-separable preferences with infinitely elastic 
labor $\left(1 / \varepsilon_{l l}=1 / \varepsilon_{c l}=1 / \varepsilon_{l c}=0\right)$, condition (16) becomes $\tau>s / \sigma$. The same necessary condition has been derived by Ghilardi and Rossi (2014) in this particular case with a CES technology.

\subsection{Linearly homogeneous preferences}

A linearly homogeneous specification is characterized by $\varepsilon_{l c}, \varepsilon_{c l}<0$ such that consumption and labor are always Edgeworth substitutes. Moreover, notice

that $\frac{1}{\varepsilon_{c c}} \frac{1}{\varepsilon_{l l}}-\frac{1}{\varepsilon_{c l}} \frac{1}{\varepsilon_{l c}}=0$ and we obtain therefore from (16) $\underline{\tau}=\frac{s}{\sigma}$. We derive the next Proposition:

Proposition 2. Under Assumptions 1-3, let $U(c, \mathcal{L} / B)$ be linearly homogeneous and $\underline{\tau}=\frac{s}{\sigma}$. There exist $\bar{\rho} \in(0,+\infty], \bar{\tau} \in(\underline{\tau}, 1)$ and $\bar{\varepsilon}_{c c}>0$ such that the NSS is locally indeterminate if and only if $\rho \in(0, \bar{\rho}), \varepsilon_{c c}<\bar{\varepsilon}_{c c}$ and $\tau \in(\underline{\tau}, \bar{\tau})$.

Proof: See Appendix 8.1.

We show that local indeterminacy easily arises under linearly homogeneous preferences for tax rates within a bounded interval. Note that in order to have $\underline{\tau}<\bar{\tau}$, we need a low enough elasticity of intertemporal substitution in consumption, i.e. $\varepsilon_{c c}<\bar{\varepsilon}_{c c}$, where $\bar{\varepsilon}_{c c}$ can be arbitrarily large when the share of consumption in total utility $\alpha$ is close to unity. Finally, the upper-bound on the elasticity of intertemporal substitution in consumption has important 
implications on the wage elasticity of labor $\varepsilon_{l l}$. It can be shown that $\varepsilon_{c c}<\bar{\varepsilon}_{c c}$ implies a sufficiently low wage elasticity of labor $\varepsilon_{l l}<\bar{\varepsilon}_{l l}$.

\subsection{Jaimovich-Rebelo preferences}

Considering now JR preferences, we show that local indeterminacy also arises for tax rates within a bounded interval. We get the following Proposition:

Proposition 3. Under Assumptions 1-3, let $U(c, \mathcal{L} / B)$ be given by (4). There is a critical value $\underline{\gamma} \in(0,1)$ such that for any given $\gamma \in(\underline{\gamma}, 1]$, there exist $\bar{\rho} \in(0,+\infty], \underline{\theta} \in(0, \gamma), \bar{\theta} \in(\underline{\theta},+\infty], \underline{\sigma} \in(s,+\infty), \underline{\tau} \in(0,1)$ and $\bar{\tau} \in(\underline{\tau}, 1)$ such that the NSS is locally indeterminate if and only if $\rho \in(0, \bar{\rho}), \theta \in(\underline{\theta}, \bar{\theta})$, $\sigma>\underline{\sigma}$ and $\tau \in(\underline{\tau}, \bar{\tau})$

Proof: See Appendix 8.2.

Since $\underline{\theta} \in(0, \gamma)$, Proposition 3 shows that indeterminacy occurs when consumption and labor are either Edgeworth substitutes or weak Edgeworth complements. More precisely, local indeterminacy is ruled out with a GHH specification characterized by the absence of income effect $(\gamma=0)$ and a strong Edgeworth-complementarity. On the contrary, with KPR preferences $(\gamma=1)$, consumption and labor are Edgeworth substitutes if $\theta<1$. In this case, the existence of a range of destabilizing tax rates is ensured provided that the elastic- 
ity of intertemporal substitution in consumption is not too large $(\theta<\bar{\theta})$. Otherwise, when consumption and labor become weak Edgeworth complements, indeterminacy may still hold but requires higher tax rates. This conclusion explains therefore the determinacy result of Linnemann (2008) since he assumes KPR preferences $(\gamma=1)$ with $\theta>1$, i.e. a strong Edgeworth-complementarity between consumption and labor.

\section{$5 \quad$ Numerical illustrations}

To illustrate our results, we use the evidence on effective tax rates on labor income given by Trabandt and Uhlig (2011) by choosing countries representative of four levels of tax rates: The U.S. (28\%) for the lowest tax rates, Germany (41\%) and France (46\%), respectively for lower-intermediate and upper-intermediate rates and Sweden (56\%) for the highest tax rates. According to the empirical literature, there is no clear agreement on the size of the elasticity of capital-labor substitution. On the first hand, the higher estimates of this elasticity belong to the range $(1.24,3.24)$ as shown in Duffy and Papageorgiou (2000) and Karagiannis et al. (2005). On the other hand, León-Ledesma et al. (2010), Klump et al. (2007, 2012) and McAdam and Willman (2013) give an interval of $(0.4,0.9)$ for lower estimates. There is also no consensus on the elasticity of intertemporal substitution in consumption. 
Several contributions provide the range $(0.2,0.8)$ (see Campbell (1999) and Kocherlakota (1996)), while Mulligan (2002), Vissing-Jorgensen and Attanasio (2003) and more recently Gruber (2013) show evidences for higher estimates with an interval $(2,3)$. Finally, Rogerson and Wallenius (2009) investigate aggregate participation in the labor market at the macro level and find that the wage elasticity of labor belongs to the range $(2.25,3)$.

From the discussion above and considering calibrations based on quaterly data, Table 1 gives the values of parameters adopted:

\begin{tabular}{cccccc}
\hline \hline$\rho$ & $\delta$ & $s$ & $\varepsilon_{c c}$ & $\varepsilon_{l l}$ & $\sigma$ \\
\hline 0.01 & 0.025 & 0.3 & {$[0.66,2.5]$} & 2.5 & {$[0.8,1.4]$} \\
\hline \hline
\end{tabular}

Table 1: Calibration of the model

Note that we consider values of the elasticity of capital-labor substitution $\sigma$ larger than the share of labor $1-s$. We do not cover the lower estimates of León-Ledesma et al. (2010) and McAdam and Willman (2013). Two reasons motivate this choice. First, these studies consider biased technological change which is not present in our model. Second, as recently documented by Piketty and Zucman (2014), the share of capital in income is increasing with respect to the capital/income ratio which implies $\sigma>1$. Although we do not reject the possibility of $\sigma<1$, assuming $\sigma>1-s$ ensures the property of an increasing capital income, $r(t) K(t)$, with respect to the stock of capital. 
The parameters $\alpha$ for the linearly homogenous preferences and $\theta, \chi$ and $\gamma$ for the JR preferences are choosen in order to match the elasticities $\varepsilon_{c c}$ and $\varepsilon_{l l}$, taking as given the estimates of tax rate of the countries considered.

When the utility function is linearly homogeneous, all countries fall in the range of indeterminacy with the exception of the U.S. when $\sigma$ is low as shown in Figure 1. Considering Proposition 2 and that $\alpha \in(0.38,0.6)$, we find a lower bound for local indeterminacy $\underline{\tau} \in(0.21,0.38)$ while the inverval of the upper bound is given by $\bar{\tau} \in(0.84,0.95)$.

\section{Insert Figure 1 here.}

With the JR formulation, our conclusions are much more contrasted as shown in Figure 2. On the first hand, when $\varepsilon_{c c}=0.66$, no country experiences indeterminacy. Note that in this case, all countries exhibit Edgeworthcomplementarity between consumption and labor since $\gamma<\theta$ holds. On the other hand, when $\varepsilon_{c c}=2.5$, indeterminacy is plausible for countries with the higher taxes. In this case, we find a critical value $\underline{\gamma} \in(0.48,0.64)$ which which implies Edgeworth-substitutability as $\theta=0.5 \cdot{ }^{4}$ Furthermore, since the values of $\gamma$ implied by our calibration satisfy $(0.89,0.97)$ when $\varepsilon_{c c}=2.5$, we find an

\footnotetext{
${ }^{4}$ The interval of $\gamma$ with $\underline{\gamma} \in(0.48,0.64)$ fits the upper estimates of Kahn and Tsoukalas (2011). Using Bayesian estimations, they report indeed a distribution of $\gamma$ with mean 0.81 and a 10-90 percentiles interval of $[0.69,0.95]$.
} 
interval of the lower bound $\underline{\tau} \in(0.29,0.5)$ while the upper bound satisfies $\bar{\tau} \in(0.78,0.81)$.

Insert Figure 2 here.

Confirming the conclusions of SGU, our numerical exercises then show that most OECD countries may experience instability for a large set of nonseparable preferences with plausible values of structural parameters. Note however that we find local indeterminacy with significantly lower tax rates than SGU. It is also worth noting that while our formulation of the balancedbudget rule implies an elasticity of the tax rate with respect to wage income equal to -1 , tax rates are not necessarily characterized by strong variations. Indeed, as shown in SGU, neither the first-order serial correlations, the contemporaneous correlations with output, nor the standard deviation relative to output of taxes, output, hours, and consumption is affected by the relative volatility of the sunspot shock or its correlation with the technology shock.

\section{Economic intuition}

To get an intuition for our results, let us start from the steady state and assume that agents expect an increase of the future tax rate. Following (7) and (10), future labor supply decreases and yields a lower interest rate. Consequently, current investment decreases and since households need to work less, 
they decrease their current labor supply below the steady state value. This generates a decline of income, leading agents to also decrease their current consumption below their steady state value, and thus a decline of the tax base that yields the government to balance its budget by increasing tax rates. It follows that the initial agents' expectations are self-fulfilling. Nevertheless one question remains: Why do expectations-driven fluctuations occur under some class of preferences while they are ruled out with others? The crucial point relies on the cross-elasticity $\varepsilon_{l c}$ that needs to be negative or weakly positive, i.e. consumption and labor need to be Edgeworth substitutes or weak Edgeworth complements.

Since capital is predetermined, we have $\dot{w} / w=-(s / \sigma) \dot{l} / l$. Taking then the derivative with respect to time of the equation that describes the consumption/leisure trade-off, indeterminacy occurs if the following equality is satisfied:

$$
\left(\frac{s}{\sigma}+\frac{1}{\varepsilon_{l l}}\right) \frac{\dot{i}}{l}-\frac{1}{\varepsilon_{l c}} \frac{\dot{c}}{c}=\frac{\dot{\lambda}}{\lambda}
$$

The decrease in interest rate involves $\dot{\lambda}>0$ since $r<\delta+\rho$. As current consumption and labor have decreased with respect to their steady state values, we conclude that self-fulfilling expectations imply $\dot{c} / c>0$ and $l / l>0$ in order to guarantee the convergence toward the long run equilibrium. Therefore, expectations will be self-fulfilling if these different effects satisfy equation (17).

As $\left(\frac{s}{\sigma}+\frac{1}{\varepsilon_{l l}}\right)$ is positive, this equation is satisfied if $1 / \varepsilon_{l c}$ is negative or positive 
but sufficiently low such that the first term on the left-hand side dominates the second one. Obviously, this is always the case with linearly homogeneous preferences since $\varepsilon_{l c}<0$. On the contrary, with JR preferences, the sign of $\varepsilon_{l c}$ is ambiguous and depends in particular on the size of $\tau$ and $\gamma$, namely

$$
\frac{1}{\varepsilon_{l c}}=\frac{(\theta-\gamma)(1+\chi)+\gamma(1-\gamma) \mathcal{C}(\tau)}{1+\chi-(1-\gamma) \mathcal{C}(\tau)}
$$

where

$$
\mathcal{C}(\tau) \equiv \frac{(1-\tau)(\delta+\rho)(1-s)}{(1-\tau)(\delta+\rho)(1-s)+s \rho}
$$

is a decreasing function with respect to $\tau$. The denominator in equation (18) being positive, the sign of $\varepsilon_{l c}$ is given by the numerator. Consider first that $\gamma>\theta$. The numerator of (18) is then negative for $\gamma$ close enough to one so that the JR utility function displays Edgeworth-substitutability and equation (17) is always satisfied. In contrast, when $\gamma<\theta$, the numerator is positive and consumption and labor are therefore Edgeworth complements. It follows that the intertemporal mechanisms described in equation (17) can be satisfied only if $1 / \varepsilon_{l c}$ is not too large. Since $\mathcal{C}$ is decreasing in $\tau$, this requires higher tax rates. Assuming $\gamma=1$ and $\theta>1$, Linneman (2008) postulates a strong complementarity between consumption and labor that prevents the existence of local indeterminacy under realistic tax rates. 


\section{Concluding comments}

This paper contributes to the debate on the destabilizing properties of balanced-budget rules. We show that, contrary to the conclusion of Linneman (2008), there exists a large set of non-separable preferences for which balanced-budget rules with labor income taxes can easily generate macroeconomic instability and expectations-driven fluctuations. We prove that the existence of self-fulfilling expectations requires Edgeworth-substitutability or weak Edgeworth-complementarity between consumption and labor. Under these conditions, an intermediate range of tax rates is destabilizing if the elasticity of intertemporal substitution in consumption is sufficiently low. We then confirm and extend the initial findings of SGU showing the plausibility of balanced-budget rules as a source of macroeconomic instability for a large sample of OECD countries.

\section{References}

[1] Campbell, John (1999) Asset prices, consumption and the business cycle. In: John B. Taylor and Michael Woodford (Eds.), Handbook of Macroeconomics, pp. 1231-1303. North-Holland, Amsterdam.

[2] Duffy, John and Chris Papageorgiou (2000) A cross-country empirical investigation of the aggregate production function specification. Journal 
of Economic Growth 5, 87-120.

[3] Ghilardi, Matteo and Raffaele Rossi (2014) Aggregate stability and balanced-budget rules. Journal of Money, Credit and Banking 46, 17851807.

[4] Greenwood, Jeremy, Zvi Hercovitz, and Gregory Huffman (1988) Investment, capacity utilization and the real business cycle. American Economic Review 78, 402-417.

[5] Gruber, Jonathan (2013) A tax-based estimate of the elasticity of intertemporal substitution. Quarterly Journal of Finance 3, 1-20.

[6] Guo, Jang-Ting and Lansing, Kevin (1998) Indeterminacy and stabilization policy. Journal of Economic Theory 82, 481-490.

[7] Guo, Jang-Ting and Sharon Harrison (2004) Balanced-budget rules and macroeconomic (in)stability. Journal of Economic Theory 119, 357-363.

[8] Jaimovich, Nir and Sergio Rebelo (2008) Can news about the future drive business cycles? American Economic Review 99, 1097-1118.

[9] Karagiannis, Giannis, Theodore Palivos and Chris Papageorgiou (2005) Variable elasticity of substitution and economic growth: theory and evidence. In: Diebold, Claude and C. Kyrtsou (Eds.), New Trends in Macroeconomics. Springer, Heidelberg. 
[10] Khan, Hashmat and John Tsoukalas (2011) Investment shocks and the comovement problem. Journal of Economic Dynamics and Control 35, $115-130$.

[11] King, Robert, Charles Plosser and Sergio Rebelo (1988) Production, growth and business cycles II: new directions. Journal of Monetary Economics $21,309-341$.

[12] Klump, Rainer, Peter McAdam and Alpo Willman (2007) The long-term success of the neoclassical growth model. Oxford Review of Economic Policy 23, 94-114.

[13] Klump, Rainer, Peter McAdam and Alpo Willman (2012) The normalized CES production function - theory and empirics. Journal of Economic Surveys 26, 769-799.

[14] Kocherlakota, Narayana (1996) The equity premium: it's still a puzzle. Journal of Economic Literature 36, 42-71.

[15] Lane, Philip (2003) The cyclical behaviour of fiscal policy: evidence from the OECD. Journal of Public Economics 87, 2661-2675.

[16] León-Ledesma, Miguel, Peter McAdam and Alpo Willman (2010) Identifying the elasticity of substitution with biased technical change. American Economic Review 100, 1330-1357. 
[17] Linnemann, Ludger (2008) Balanced budget rules and macroeconomic stability with non-separable utility. Journal of Macroeconomics 30, 199215

[18] MacAdam, Peter and Alpo Willman (2013) Medium run redux. Macroeconomic Dynamics $17,695-727$.

[19] Mulligan, Casey (2002) Capital interest and aggregate intertemporal substitution. NBER Working Paper, 9373.

[20] Piketty, Thomas and Gabriel Zucman (2014) Capital is back: wealthincome ratio in rich countries 1700-2010. Quaterly Journal of Economics $129,1255-1310$.

[21] Rogerson, Richard and Johanna Wallenius (2009) Micro and macro elasticities in a life cycle model with taxes. Journal of Economic Theory 144, $2277-2292$.

[22] Schaechter, Andrea, Tidiane Kinda, Nina Budina and Anke Weber (2012) Fiscal rules in response to the crisis. IMF Working Paper, 12/187.

[23] Schmitt-Grohé, Stephanie and Martin Uribe (1997) Balanced-budget rules, distortionary taxes, and aggregate instability. Journal of Political Economy 105, 976-1000. 
[24] Trabandt, Mathias and Harald Uhlig (2011) The Laffer curve revisited. Journal of Monetary Economics 58, 305-327.

[25] Vissing-Jorgensen, Annette and Orazio Attanasio (2003) Stock-market participation, intertemporal substitution and risk aversion. American Economic Review Papers and Proceedings 93, 383-391.

\section{Appendix}

\subsection{Proof of Proposition 2}

Using the general expressions for the Trace and the Determinant as given in Section 3 of the Technical Appendix, the Trace and the Determinant with linearly homogeneous preferences are given by:

$$
\mathcal{T}=\rho-\frac{(\rho+\delta)(1-s) \tau}{\sigma \tau-s}
$$

and

$$
\mathcal{D}=\frac{(1-\tau)(\delta+\rho)(1-s)}{(1-\alpha) s \sigma(\sigma \tau-s)} P(\tau)
$$

with

$P(\tau)=[(\rho+\delta)(1-s)+s \rho]+\frac{\alpha}{1-\alpha}(1-\tau)(\rho+\delta)(1-s)-\frac{\tau[(1-\tau)(\rho+\delta)(1-s)+s \rho](1-\alpha) \varepsilon_{c c}}{(1-\tau)}$

From the trace, we derive the lower bound on tax rate as given by (16):

$$
\underline{\tau}=\frac{s}{\sigma}
$$


Under this condition, we conclude that $\mathcal{T}<0$ when:

$$
\begin{array}{ll}
\text { i) } \rho<\bar{\rho}=\frac{\delta(1-s) \tau}{\sigma \tau-s-(1-s) \tau} & \text { if } \sigma \tau-s-(1-s) \tau>0 \\
\text { ii) } \rho \in(0,+\infty) & \text { otherwise }
\end{array}
$$

Moreover, we derive:

$$
\frac{\partial P(\tau)}{\partial \tau}=-\frac{\alpha}{1-\alpha}(\rho+\delta)(1-s)-\frac{(1-\alpha) \varepsilon_{c c}}{(1-\tau)^{2}}\left[(1-\tau)^{2}(\rho+\delta)(1-s)+s \rho\right]<0
$$

The polynomial $P(\tau)$ is positive when $\tau=0$ and negative when $\tau=1$. Since $P(\tau)$ is monotonically decreasing in $\tau$, there exists therefore a unique solution $\bar{\tau} \in(0,1)$ such that $P(\tau)>0$ if $\tau<\bar{\tau}$. Since the denominator is positive when $\tau>\underline{\tau}$, the determinant is positive if and only if $\tau \in(\underline{\tau}, \bar{\tau})$. We finally need to check that $\underline{\tau}<\bar{\tau}$. Substituting $\underline{\tau}=\frac{s}{\sigma}$ into $P(\tau)$, the interval $(\underline{\tau}, \bar{\tau})$ is non-empty if and only if $P(\underline{\tau})>0$, i.e. $\varepsilon_{c c}$ is low enough such that:

$$
\varepsilon_{c c}<\bar{\varepsilon}_{c c}=\frac{(1-s / \sigma)\left[(\rho+\delta)(1-s)+s \rho+(1-s / \sigma)(\rho+\delta)(1-s) \frac{\alpha}{(1-\alpha)}\right]}{(1-\alpha) \frac{s}{\sigma}[(1-s / \sigma)(\rho+\delta)(1-s)+s \rho]}
$$

\subsection{Proof of Proposition 3}

Using the general expressions for the Trace and the Determinant as given in Section 3 of the Technical Appendix, the Trace and the Determinant with JR preferences are given by:

$$
\mathcal{T}=\rho-\frac{(\rho+\delta)(1-s) \tau}{\sigma \tau-s-(1-\tau) \sigma \varepsilon_{c c}\left[\frac{1}{\varepsilon_{c c}} \frac{1}{\varepsilon_{l l}}-\frac{1}{\varepsilon_{c l}} \frac{1}{\varepsilon_{l c}}\right]}
$$

and 


$$
\mathcal{D}=\frac{(\rho+\delta)(1-s) \varepsilon_{c c}\left[\frac{\gamma(1-\tau)[1+\chi-(1-\gamma) \mathcal{C}(\tau)]}{1+\chi}[(\rho+\delta)(1-s)+s \rho]+[(1-\tau)(\rho+\delta)(1-s)+s \rho][\gamma(1-\tau) \mathcal{C}(\tau)+\chi-\tau(1+\chi)]\right]}{s \sigma\left[\sigma \tau-s-(1-\tau) \sigma \varepsilon_{c c}\left[\frac{1}{\varepsilon_{c c}} \frac{1}{\varepsilon_{l l}}-\frac{1}{\varepsilon_{c l}} \frac{1}{\varepsilon_{l c}}\right]\right]}
$$

with

$$
\begin{aligned}
\varepsilon_{c c}\left[\frac{1}{\varepsilon_{c c}} \frac{1}{\varepsilon_{l l}}-\frac{1}{\varepsilon_{c l}} \frac{1}{\varepsilon_{l c}}\right] & =\frac{\theta(1+\chi)^{2}\left[\chi+\gamma \mathcal{C}(\tau)\left(2-\frac{(1-\gamma) \mathcal{C}(\tau)}{1+\chi}\right)\right]-\gamma \mathcal{C}(\tau)(\gamma+\chi)[1+\chi-(1-\gamma) \mathcal{C}(\tau)]}{\theta(1+\chi)^{2}-\gamma(1-\gamma) \mathcal{C}(\tau)[1+\chi-(1-\gamma) \mathcal{C}(\tau)]} \\
\frac{1}{\varepsilon_{c c}} & =\theta \frac{1+\chi}{1+\chi-(1-\gamma) \mathcal{C}(\tau)}-\gamma(1-\gamma) \frac{\mathcal{C}(\tau)}{1+\chi}
\end{aligned}
$$

and

$$
\mathcal{C}(\tau) \equiv \frac{(1-\tau)(\delta+\rho)(1-s)}{(1-\tau)(\delta+\rho)(1-s)+s \rho}
$$

Note that local concavity of the utility fonction is ensured when $\frac{1}{\varepsilon_{c c}} \geq 0$ and $\frac{1}{\varepsilon_{c c}} \frac{1}{\varepsilon_{l l}}-\frac{1}{\varepsilon_{c l}} \frac{1}{\varepsilon_{l c}} \geq 0$. These two inequalities are satisfied if and only if:

$$
\theta \geq \underline{\theta}(\tau, \gamma, \chi) \equiv \frac{\gamma \mathcal{C}(\tau)(\gamma+\chi)(1+\chi-(1-\gamma) \mathcal{C}(\tau))}{(1+\chi)^{2}\left[\chi+\gamma \mathcal{C}(\tau)\left(2-\frac{(1-\gamma) \mathcal{C}(\tau)}{1+\chi}\right)\right]}
$$

Note that Edgeworth-substitutability is possible if $\gamma \geq \underline{\theta}(\tau, \gamma, \chi)$. This inequality is satisfied when $\gamma=0$ and $\gamma=1$. It follows that it will be satisfied for any $\gamma \in(0,1)$ if

$$
1 \geq \frac{\mathcal{C}(\tau)(\gamma+\chi)(1+\chi-(1-\gamma) \mathcal{C}(\tau))}{(1+\chi)^{2}\left[\chi+\gamma \mathcal{C}(\tau)\left(2-\frac{(1-\gamma) \mathcal{C}(\tau)}{1+\chi}\right)\right]}
$$

Straightforward computations show that this inequality holds for any $\gamma \in$ $(0,1)$

We now study the indeterminacy conditions given equation (21) is satisfied. When $\gamma=0$ (GHH case), we get:

$$
\mathcal{T}=\rho-\frac{\tau(\rho+\delta)(1-s)}{\sigma \tau-s-(1-\tau) \sigma \chi}
$$




$$
\mathcal{D}=\frac{(\delta+\rho)(1-s) \varepsilon_{c c}[(1-\tau)(\rho+\delta)(1-s)+s \rho]}{\sigma s[\sigma \tau-s-\sigma(1-\tau) \chi]}[\chi-\tau(1+\chi)]
$$

From (22), a necessary condition for $\mathcal{T}<0$ is $\tau>\underline{\tau}^{0}$ with:

$$
\underline{\tau}^{0}=\frac{\frac{s}{\sigma}+\chi}{1+\chi}
$$

In equation (23), the condition $\tau>\underline{\tau}^{0}$ implies a positive denominator. The sign of the determinant is therefore determined by the second factor of (23), i.e. $\chi-\tau(1+\chi)$. This expression is positive if and only if $\tau<\bar{\tau}^{0}$ with:

$$
\bar{\tau}^{0}=\frac{\chi}{1+\chi}
$$

which is lower than $\underline{\tau}^{0}$. Since $\bar{\tau}^{0}<\underline{\tau}^{0}$, indeterminacy is therefore ruled out.

When $\gamma=1$ (KPR case), we now get:

$$
\mathcal{T}=\rho-\frac{(\rho+\delta)(1-s) \tau}{\sigma G(\tau)}
$$

and

$$
\mathcal{D}=\frac{(\rho+\delta)(1-s) \varepsilon_{c c}}{s \sigma G(\tau)} P(\tau)
$$

with

$$
G(\tau)=\tau-\frac{s}{\sigma}-(1-\tau) \chi-(1-\tau) \mathcal{C}(\tau)\left(2-\frac{1}{\theta}\right)
$$

and

$$
\begin{gathered}
P(\tau)=[(1-\tau)(\rho+\delta)(1-s)+s \rho][\chi-\tau(1+\chi)] \\
+(1-\tau)^{2}(\rho+\delta)(1-s)+(1-\tau)[(\rho+\delta)(1-s)+s \rho]
\end{gathered}
$$

Indeterminacy requires that both $G(\tau)$ and $P(\tau)$ are positive. In order to get $G(\tau)>0$, one can use Lemma 1 . The lower bound $\underline{\tau}^{1}$ as given in (16) is implicitly given by $\underline{\tau}^{1}=h\left(\underline{\tau}^{1}\right)$ with: 


$$
h(\tau)=\frac{\frac{s}{\sigma}+\chi+\left(2-\frac{1}{\theta}\right) \mathcal{C}(\tau)}{1+\chi+\left(2-\frac{1}{\theta}\right) \mathcal{C}(\tau)}
$$

We derive that $h(0)>0$ while $h(1)=\frac{\frac{s}{\sigma}+\chi}{1+\chi}<1$. There exists therefore $\underline{\tau}^{1} \in(0,1)$ such that $G(\tau)>0$ if and only if $\tau>\underline{\tau}^{1}$. Moreover, considering the expression of $G(\tau)$, we can show that $\underline{\tau}^{1}$ is unique. We directly observe that when $\rho$ tends to zero, the trace is negative if $\tau>\underline{\tau}^{1}$. There exists therefore $\bar{\rho}^{1}>0$ such that $\mathcal{T}<0$ if and only if $\tau>\underline{\tau}^{1}$ and $\rho \in\left(0, \bar{\rho}^{1}\right)$.

For the determinant, $P(\tau)$ is positive when $\tau=0$ and negative when $\tau=1$. Since $P(\tau)$ is strictly decreasing in $\tau \in(0,1)$, there exists therefore a unique $\bar{\tau}^{1} \in(0,1)$ such that $P(\tau)>0$ if and only if $\tau<\bar{\tau}^{1}$. The determinant is therefore positive if and only if $\tau \in\left(\underline{\tau}^{1}, \bar{\tau}^{1}\right)$. The condition $\underline{\tau}^{1}<\bar{\tau}^{1}$ is satisfied if and only if $P\left(\underline{\tau}^{1}\right)>0$. Note that substituting $\theta=\underline{\theta}^{1} \equiv \underline{\theta}(\tau, 1, \chi)$ in $(24)$, we get:

$$
\underline{\tau}^{1}=\frac{s}{\sigma}
$$

When $\mathrm{P}(\tau)$ is evaluated at $\tau=\frac{s}{\sigma}$, we obtain:

$$
\begin{aligned}
P\left(\frac{s}{\sigma}\right) & =\left[\left(1-\frac{s}{\sigma}\right)(\rho+\delta)(1-s)+s \rho\right]\left[\chi-\frac{s}{\sigma}(1+\chi)\right]+\left(1-\frac{s}{\sigma}\right)^{2}(\rho+\delta)(1-s) \\
& +\left(1-\frac{s}{\sigma}\right)[(\rho+\delta)(1-s)+s \rho]
\end{aligned}
$$

On the one hand, if $\sigma=s$, we get $P\left(\frac{s}{\sigma}\right)=-s \rho<0$. On the other hand, when $\sigma$ tends to $+\infty, P\left(\frac{s}{\sigma}\right)$ is positive. There exist therefore $\underline{\sigma}^{1} \in(s,+\infty)$ such that $\underline{\tau}^{1}<\bar{\tau}^{1}$ if and only if $\theta=\underline{\theta}^{1}$ and $\sigma>\underline{\sigma}^{1}$. By a continuity argument, there exists therefore $\bar{\theta}^{1} \in\left(\underline{\theta}^{1},+\infty\right]$ and $\underline{\sigma}^{1} \in(s,+\infty)$ such that $\underline{\tau}^{1}<\bar{\tau}^{1}$ if and only if $\theta \in\left[\underline{\theta}^{1}, \bar{\theta}^{1}\right)$ and $\sigma>\underline{\sigma}^{1}$. 
Given that indeterminacy occurs when $\gamma=1, \tau \in\left(\underline{\tau}^{1}, \bar{\tau}^{1}\right), \theta \in\left[\underline{\theta}^{1}, \bar{\theta}^{1}\right)$, $\rho \in\left(0, \bar{\rho}^{1}\right)$ and $\sigma>\underline{\sigma}^{1}$, but is ruled out when $\gamma=0$, there exists therefore $\underline{\gamma} \in(0,1)$ such that for any $\gamma \in(\underline{\gamma}, 1]$, there exist $\underline{\tau} \in(0,1), \bar{\tau} \in(\underline{\tau}, 1)$, $\bar{\rho} \in(0,+\infty], \bar{\theta} \in(\underline{\theta},+\infty]$ and $\underline{\sigma} \in(s,+\infty)$ such that the NSS is locally indeterminate if and only if $\tau \in(\underline{\tau}, \bar{\tau}), \theta \in[\underline{\theta}, \bar{\theta}), \rho \in(0, \bar{\rho})$ and $\sigma>\underline{\sigma}$.
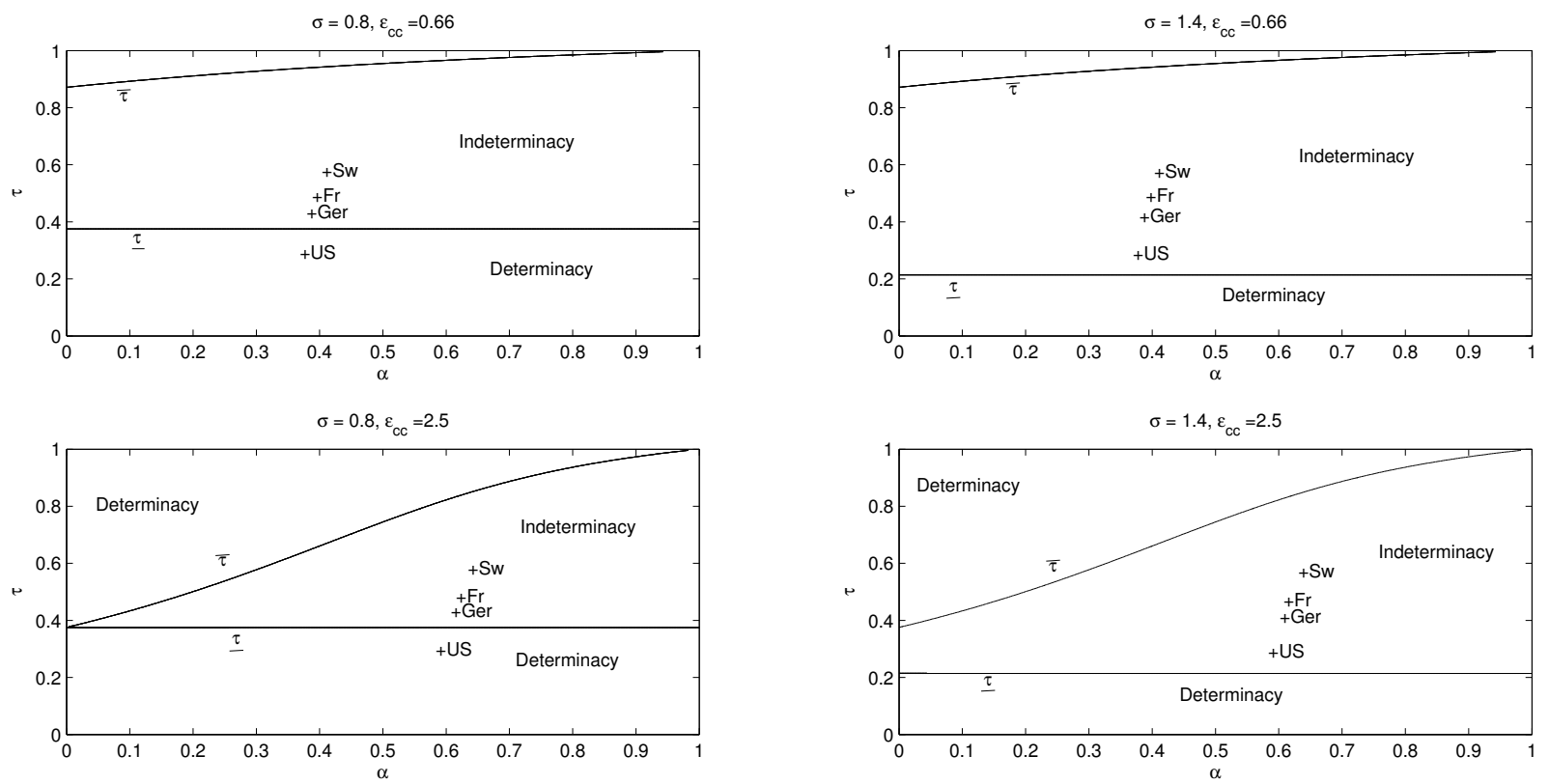

Figure 1: Destabilizing tax rates in the case with linearly homogeneous preferences. 

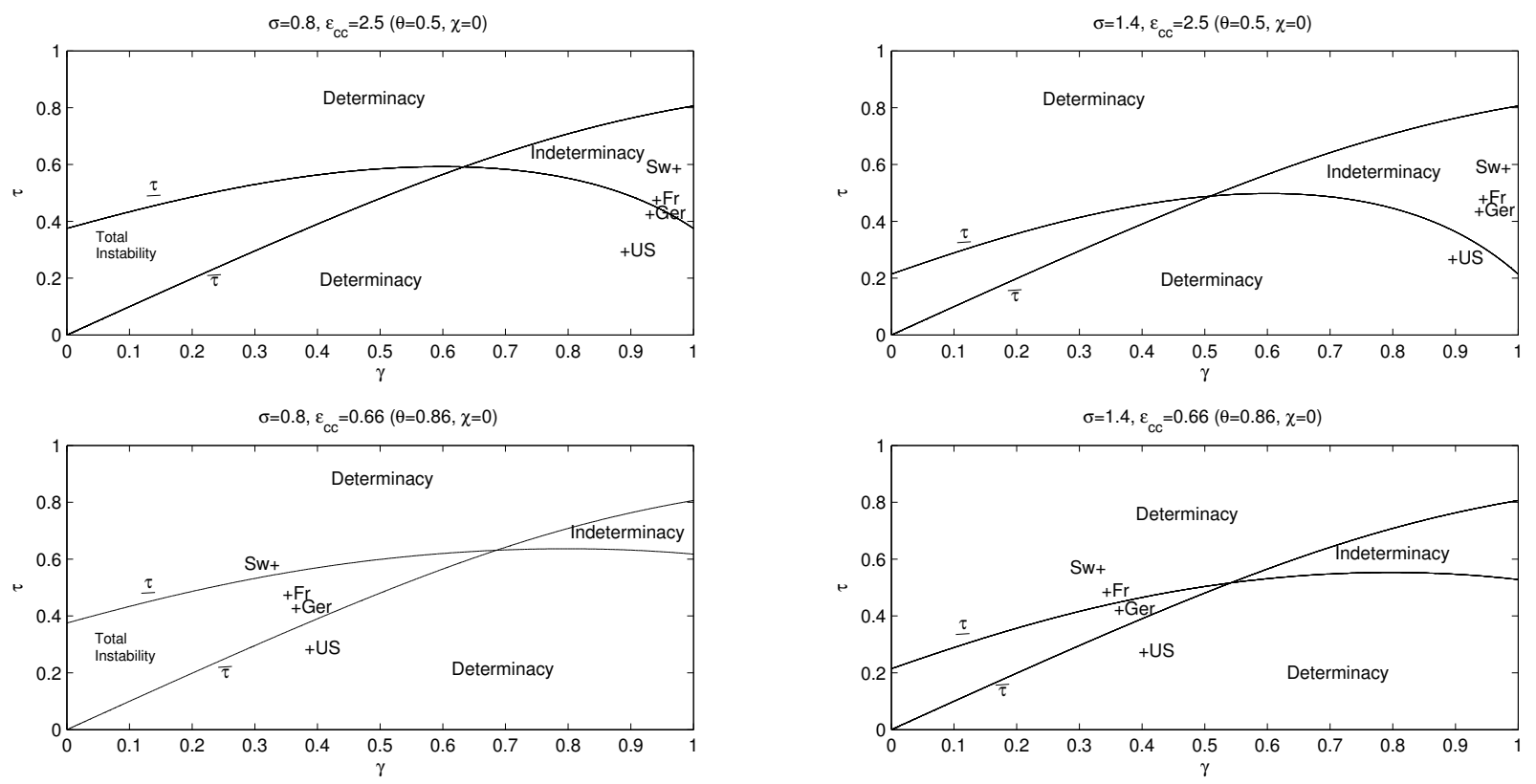

Figure 2: Destabilizing tax rates in the case with Jaimovich-Rebelo preferences. 